doi: https://doi.org/10.15407/dopovidi2018.01.022

УдК 539.3

\title{
А.М. Багно
}

Институт механики им. С.П. Тимошенко НАН Украины, Киев

E-mail: alexbag2016@gmail.com

\section{О локализации поверхностных волн в слое идеальной сжимаемой жидкости, взаимодействующем с упругим полупространством}

Представлено академиком НАН Украины А.Н. Гузем

На основании трехмерных линейных уравнений классической теории упругости для твердого тела и линеаризованных уравнений Эйлера для жидкой среды исследовано распространение квазилэмбовских волн в системе: слой идеальной сжимаемой жидкости - упругое полупространство. Построены дисперсионные кривые для нормальных волн в широком диапазоне частот. Проанализировано влияние толщины жидкого слоя на дисперсию фазовых скоростей квазилэмбовских мод в гидроупругом волноводе. Исследованы свойства локализации поверхностных волн в упруго-жидкостных волноводах. Числовые результаты приведены в виде графиков и дан их анализ.

Ключевые слова: дисперсия волн, фазовая скорость, упругое полупространство, слой идеальной сжимаемой жидкости, квазилэмбовские моды, локализация поверхностных волн.

Задача о распространении нормальных волн в слое жидкости, взаимодействующем с упругим полупространством, принадлежит к классическим задачам механики. Ей посвящена обширная библиография. Вместе с тем имеются еще вопросы, изученные недостаточно полно и до настоящего времени остающиеся актуальными. Обзор работ и анализ результатов, полученных в рамках классической теории упругости и модели идеальной сжимаемой жидкости, а также с привлечением более общих моделей твердых и жидких сред, приведены в [1-4]. Значительное практическое использование акустических волн ставит задачу изучения дисперсионных характеристик и свойств локализации квазилэмбовских мод в гидроупругом волноводе, состоящем из упругого полупространства и слоя идеальной сжимаемой жидкости, в широком диапазоне частот, охватывающем как длинноволновую, так и коротковолновую части спектра для толщин жидкого слоя соизмеримых с длиной волны. В настоящей работе для анализа дисперсионных характеристик и локализации нормальных волн в системе жидкий слой - упругое полупространство в широком интервале частот используются трехмерные линеаризованные уравнения Эйлера для жидкости и линейные уравнения классической теории упругости для твердого тела. При этом предполагается, что жидкость находится в состоянии покоя. В качестве подхода выбраны постановки задач (C) A.М. Багно, 2018 
и метод, основанные на применении представлений общих решений уравнений движения идеальной сжимаемой жидкости и упругого тела, предложенные в работах [5-9].

Постановка задачи. Рассмотрим задачу о распространении нормальных волн в гидроупругой системе, состоящей из упругого полупространства и жидкого слоя. Решение получим с привлечением трехмерных линейных уравнений классической теории упругости для твердого тела и линеаризованных уравнений Эйлера для жидкости, находящейся в состоянии покоя. В рамках принятых моделей основные соотношения для системы изотропное упругое тело - идеальная сжимаемая жидкость будут иметь вид [5-9]:

1) упругое тело

$\mu \Delta \boldsymbol{u}+(\lambda+\mu) \nabla(\nabla \cdot \boldsymbol{u})-\rho \frac{\partial^{2} \boldsymbol{u}}{\partial t^{2}}=0 ; \sigma_{i j}=\mu\left(\frac{\partial u_{i}}{\partial u_{j}}+\frac{\partial u_{j}}{\partial u_{i}}\right)+\lambda \delta_{i j} \nabla \cdot \boldsymbol{u}, z_{k} \in V_{1} ;$

2) идеальная сжимаемая жидкость

$\frac{\partial v}{\partial t}+\frac{1}{\rho_{0}} \nabla p=0, z_{k} \in V_{2}$

$\frac{1}{\rho_{0}} \frac{\partial \rho^{*}}{\partial t}+\nabla \cdot v=0 ; \frac{\partial p}{\partial \rho^{*}}=a_{0}^{2} ; a_{0}=$ const,$z_{k} \in V_{2}$;

$P_{i j}=-\delta_{i j} p$.

При этом специфику взаимодействия упругих и жидких сред отражают динамические $\sigma_{i j}=P_{i j}, z_{k} \in S$ и кинематические $\frac{\partial \boldsymbol{u}}{\partial t}=v, z_{k} \in S$ граничные условия, задаваемые на поверхности контакта твердых тел и жидкости $S$.

Здесь введены следующие обозначения: $u_{i}$ - компоненты вектора перемещений твердого тела $\boldsymbol{u} ; \rho-$ плотность материала упругого полупространства; $\lambda$ и $\mu-$ константы Ляме материала упругого тела; $v_{i}-$ составляющие вектора возмущений скорости жидкости $\boldsymbol{v}$; $\rho^{*}$ и $p$ - возмущения плотности и давления в жидкости; $\rho_{0}$ и $a_{0}-$ плотность и скорость звука в жидкости в состоянии покоя; $P_{i j}$ и $\sigma_{i j}-$ составляющие напряжений, соответственно, в жидкости и упругом теле; $V_{1}$ и $V_{2}$ - объемы занимаемые, соответственно, упругим телом и жидкостью; $S$ - поверхность контакта упругой и жидкой сред.

Далее предположим, что изотропное упругое полупространство занимает объем: $-\infty<z_{1}<\infty,-\infty<z_{2} \leqslant 0,-\infty<z_{3}<\infty$ и контактирует со слоем идеальной сжимаемой жидкости, заполняющей объем: $-\infty<z_{1}<\infty, 0 \leqslant z_{2} \leqslant h,-\infty<z_{3}<\infty$. Примем, что внешние силы, действующие на указанные среды, распределены равномерно вдоль оси $о z_{3}$. В этом случае задача является плоской и можно ограничиться изучением процесса распространения волн в плоскости $о z_{1} z_{2}$.

Воспользуемся представлениями общих решений уравнений движения упругих и жидких сред, полученными в работах [5-9]. Для плоского случая, который рассматривается далее, общие решения будут такими:

$$
u_{1}=-\frac{\partial^{2} \chi_{1}}{\partial z_{1} \partial z_{2}} ; u_{2}=\left(\frac{\lambda+2 \mu}{\lambda+\mu} \frac{\partial^{2}}{\partial z_{1}^{2}}+\frac{\mu}{\lambda+\mu} \frac{\partial^{2}}{\partial z_{2}^{2}}-\frac{\rho}{\lambda+\mu} \frac{\partial^{2}}{\partial t^{2}}\right) \chi_{1} ; v_{1}=\frac{\partial^{2} \chi_{2}}{\partial z_{1} \partial t} ; v_{2}=\frac{\partial^{2} \chi_{2}}{\partial z_{2} \partial t}
$$


где введенные функции $\chi_{1}$ и $\chi_{2}$ удовлетворяют уравнениям

$$
\begin{aligned}
& {\left[\left(\frac{\partial^{2}}{\partial z_{1}^{2}}+\frac{\mu}{\lambda+2 \mu} \frac{\partial^{2}}{\partial z_{2}^{2}}-\frac{\rho}{\lambda+2 \mu} \frac{\partial^{2}}{\partial t^{2}}\right)\left(\frac{\partial^{2}}{\partial z_{1}^{2}}+\frac{\lambda+2 \mu}{\mu} \frac{\partial^{2}}{\partial z_{2}^{2}}-\frac{\rho}{\mu} \frac{\partial^{2}}{\partial t^{2}}\right)-\frac{(\lambda+\mu)^{2}}{\mu(\lambda+2 \mu)} \frac{\partial^{4}}{\partial z_{1}^{2} \partial z_{2}^{2}}\right] \chi_{1}=0 ;} \\
& {\left[\frac{\partial^{2}}{\partial z_{1}^{2}}+\frac{\partial^{2}}{\partial z_{2}^{2}}-\frac{1}{a_{0}^{2}} \frac{\partial^{2}}{\partial t^{2}}\right] \chi_{2}=0 .}
\end{aligned}
$$

Данная задача характеризуется следующими динамическими и кинематическими граничными условиями:

$$
\left.\sigma_{12}\right|_{z_{2}=0}=0 ;\left.\sigma_{22}\right|_{z_{2}=0}=\left.P_{22}\right|_{z_{2}=0} ;\left.P_{22}\right|_{z_{2}=h}=0 ;\left.v_{2}\right|_{z_{2}=0}=\left.\frac{\partial u_{2}}{\partial t}\right|_{z_{2}=0} .
$$

Далее параметры, характеризующие процесс распространения волн, разыскиваем в классе бегущих волн и выбираем в виде

$$
\chi_{j}=X_{j}\left(z_{2}\right) \exp \left[i\left(k z_{1}-\omega t\right)\right](j=1,2),
$$

где $k-$ волновое число; $\omega-$ круговая частота; $i^{2}=-1$.

Заметим, что выбранный в работе класс гармонических волн, являясь наиболее простым и удобным в теоретических исследованиях, не ограничивает общности полученных результатов, поскольку линейная волна произвольной формы, как известно, может быть представлена набором гармонических составляющих. Далее решаем две задачи Штурма Лиувилля на собственные значения для уравнений движения жидкости и упругого тела, а также определяем соответствующие собственные функции. После подстановки решений в граничные условия (1) получаем систему линейных однородных алгебраических уравнений относительно постоянных интегрирования. Исходя из условия существования нетривиального решения, получаем дисперсионное уравнение

$$
\operatorname{det}\left\|e_{l m}\left(c, \lambda, \mu, \rho, \rho_{0}, a_{0}, \omega h / c_{s}\right)\right\|=0 \quad(l, m=\overline{1,4}),
$$

где $c$ - фазовая скорость нормальных волн; $c_{s}\left(c_{s}^{2}=\mu / \rho\right)-$ скорость волны сдвига в упругом теле; $\mu$ - модуль сдвига материала упругого полупространства; $h$ - толщина слоя жидкости.

Как известно, в неограниченном сжимаемом упругом теле существуют продольная и сдвиговая волны. В идеальной сжимаемой жидкой среде распространяется только продольная волна. Именно эти волны, взаимодействуя между собой на свободных граничных поверхностях, а также на поверхности контакта сред, порождают сложное волновое поле в гидроупругой системе.

Отметим, что полученное дисперсионное уравнение (2) является наиболее общим и из него можно получить соотношения для ряда частных случаев. В частности, если $a_{0}$ устремить к бесконечности, то (2) переходит в уравнение для определения параметров мод в случае взаимодействия с идеальной несжимаемой жидкостью. Если положить $\rho_{0}=0$, получим соотношение для определения скоростей поверхностных волн Рэлея $[1,4,5]$. При $\rho_{0} \neq 0$ и $h \rightarrow \infty$ равенство (2) перейдет в уравнение Стоунли - Шольте $[1,4,5]$.

Числовые результаты и их анализ. В дальнейшем дисперсионное уравнение (2) решаем численно. При этом расчеты проводим для двух гидроупругих систем, состоящих из ре- 

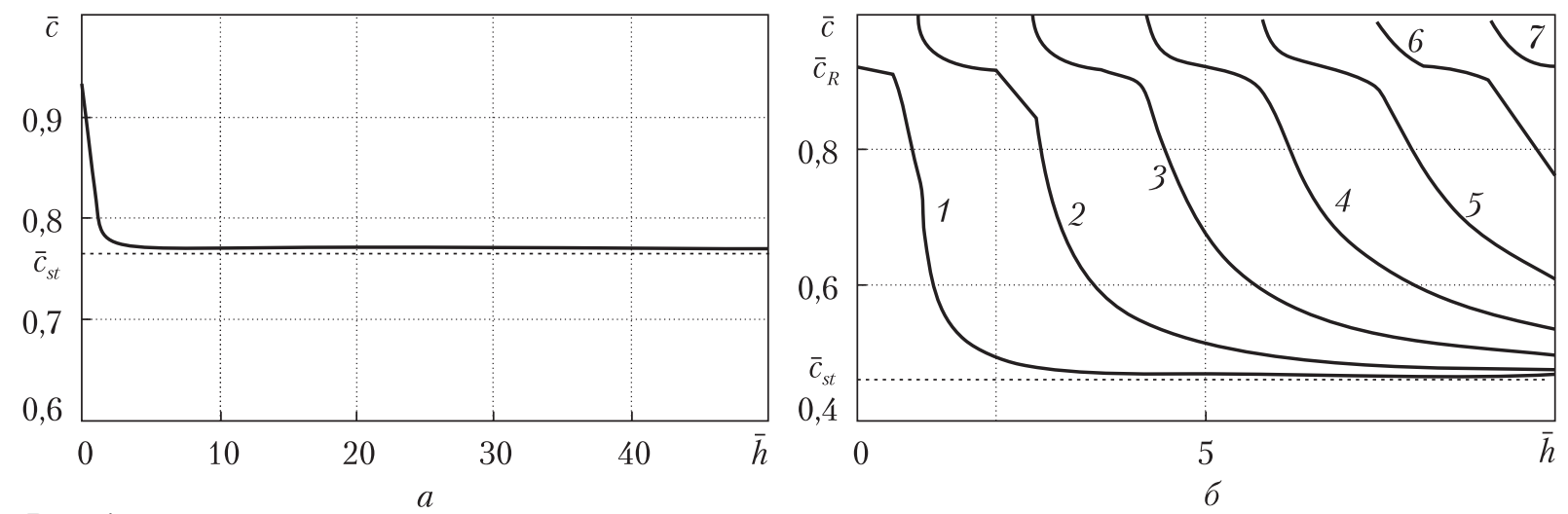

Puc. 1
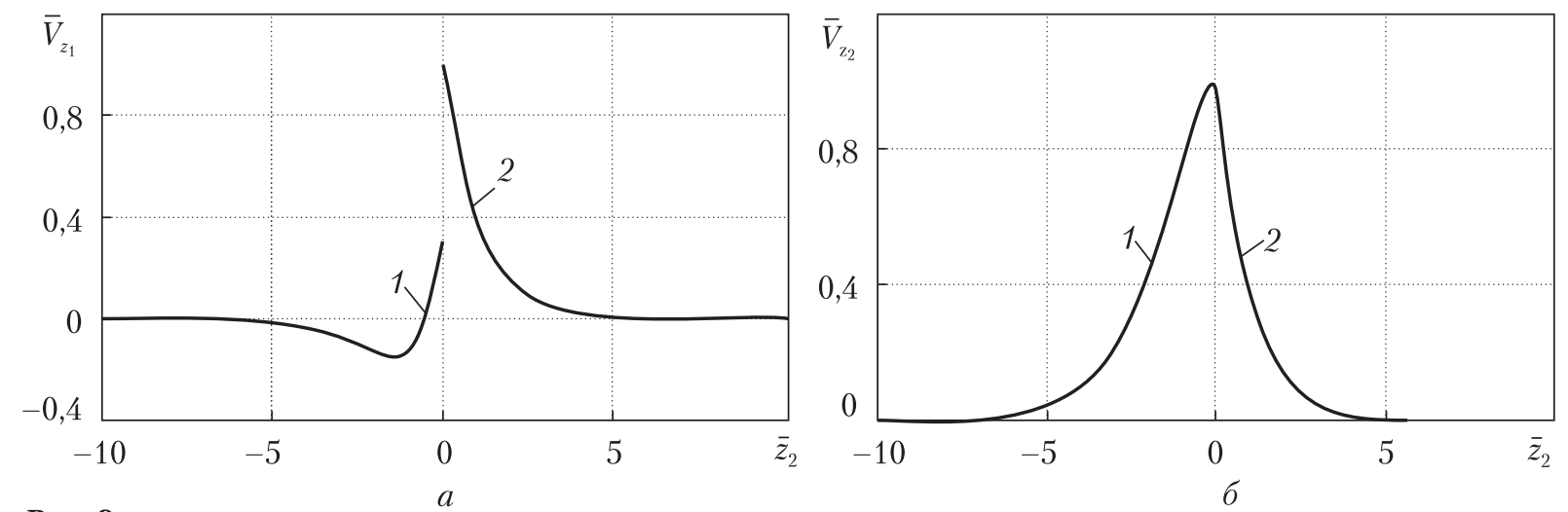

Puc. 2

альных упругих тел и жидкости. Первая состоит из органического стекла и воды. Она характеризуется следующими параметрами: упругое тело $-\rho=1160 \mathrm{\kappa г} / \mathrm{m}^{3}, \lambda=3,96 \cdot 10^{9}$ Па, $\mu=1,86 \cdot 10^{9}$ Па; жидкость - $\rho_{0}=1000 \mathrm{\kappa г} / \mathrm{m}^{3}, a_{0}=1459,5 \mathrm{~m} / \mathrm{c}, \bar{a}_{0}=a_{0} / c_{s}=1,152595$. У этого волновода материал упругого тела (органическое стекло) является жестким. Вторая представляет собой волновод из стали и воды. При этом параметры выбираем такими: упругое тело $-\rho=7800 \mathrm{\kappa г} / \mathrm{m}^{3}, \lambda=9,26 \cdot 10^{10}$ Па, $\mu=7,75 \cdot 10^{10}$ Па; жидкость $-\rho_{0}=1000 \mathrm{\kappa г} / \mathrm{m}^{3}$, $a_{0}=1459,5 \mathrm{~m} / \mathrm{c}, \bar{a}_{0}=a_{0} / c_{s}=0,463021$. Этот волновод отличается тем, что материал упругого тела (сталь) относится к разряду более жестких, чем органическое стекло.

Результаты вычислений представлены на рис. 1-3. При этом на рис. 1, $a$ и рис. 2 представлены графики для упругого полупространства из органического стекла (менее жесткий материал) и воды ( $\left.\bar{a}_{0}=1,152595\right)$. На рис. $1, \sigma$ и 3 приведены графики для упругого полупространства из стали (более жесткий материал) и слоя воды.

На рис. 1 изображены дисперсионные кривые для гидроупругих волноводов, отражающие зависимости безразмерных величин фазовых скоростей квазилэмбовских мод $\bar{c}$ $\left(\bar{c}=c / c_{s}\right)$ от безразмерной величины толщины слоя жидкости (частоты) $\bar{h}\left(\bar{h}=\omega h / c_{s}\right)$.

На рисунках для наглядности штриховыми линиями отмечены асимптотики, к которым стремятся фазовые скорости первых мод при возрастании толщины (частоты) жидкого слоя $\bar{h}$. 

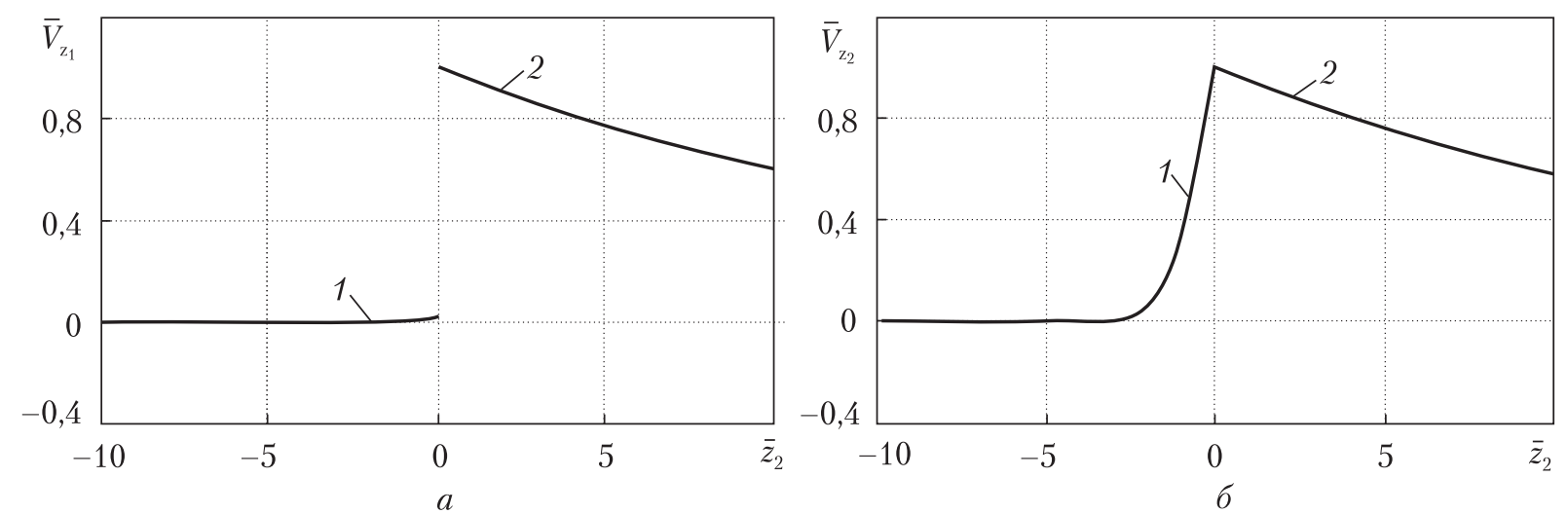

Puc. 3

Графики, представленные на рис. 2, отражают распределение смещений для гидроупругого волновода, состоящего из органического стекла $\left(\bar{z}_{2} \leqslant 0\right)$ и воды $\left(0 \leqslant \bar{z}_{2} \leqslant \bar{h}\right)$. На них приведены зависимости нормированных величин продольных $V_{\bar{z}_{1}}$ (рис. $\left.2, a\right)$ и поперечных $\bar{V}_{\bar{z}_{2}}$ (рис. $\left.2, \sigma\right)$ смещений (скоростей $\partial u_{i} / \partial t$ и $v_{i}$ ) от безразмерной поперечной координаты $\bar{z}_{2}$ для поверхностной волны 1 (рис. $1, a$ ). При нормировании величины указанных смещений отнесены к их наибольшим значениям $\left|\bar{V}_{z_{1}} \max \right|$ и $\left|\bar{V}_{z_{2}} \max \right|$.

Аналогичные зависимости для гидроупругой системы сталь - вода приведены на рис. 3. Заметим, что разрыв продольных смещений в упругом теле (кривая 1) и в жидкости (кривая 2) на границе контакта сред ( $\bar{z}_{2}=0$ ) (рис. $2, a$ и $3, a$ ) обусловлен невязкостью (идеальностью) жидкости. Кроме того, графики, приведенные на рис. 2 и 3 , получены для первой низшей квазиповерхностной моды 1 в коротковолновой части спектра при частоте (толщине) $\bar{h}=100$.

Анализ числовых результатов. Из графика, приведенного на рис. 1, $a$, следует, что скорость поверхностной волны в упругом полупространстве из органического стекла (менее жесткий материал), взаимодействующем со слоем воды, изменяется от скорости волны Рэлея $\bar{c}_{R}\left(\bar{c}_{R}=0,933557\right)$ при $\bar{h} \rightarrow 0$ до скорости волны Стоунли - Шольте $\bar{c}_{s t}\left(\bar{c}_{s t}=0,7717101\right)$ при $\bar{h} \rightarrow \infty$. Вычисления показали, что при $\bar{h}=100$ фазовая скорость этой моды лишь незначительно отличается от скорости волны Стоунли и поэтому ее можно считать поверхностной. Из графиков распределения амплитуд смещений (скоростей), представленных на рис. 2, видно, что движения этой моды при $\bar{h}=100$ происходят как в упругом теле, так и в жидкости. При этом глубина проникновения ее в упругое полупространство больше, чем в жидкость.

Рис. 1, 6 характеризует поведение дисперсионных кривых для системы упругое полупространство из стали (более жесткий материал) - слой воды. Как видно из графиков, фазовая скорость первой моды изменяется от скорости волны Рэлея $\bar{c}_{R}\left(\bar{c}_{R}=0,923007\right)$ при $\bar{h} \rightarrow 0$ до скорости волны Стоунли - Шольте $\bar{c}_{s t}\left(\bar{c}_{s t}=0,462886\right)$ при $\bar{h} \rightarrow \infty$. Скорости мод высокого порядка при частотах их зарождения равны скорости волны сдвига в упругом полупространстве $\bar{c}_{s}$ и в дальнейшем с увеличением толщины жидкого слоя (частоты) $\bar{h}$ стремятся к скорости волны звука в жидкости $\bar{a}_{0}\left(\bar{a}_{0}=0,463021\right)$. Как и в предыдущем случае, проведенные расчеты показали, что при $\bar{h}=100$ скорость квазиповерхностной моды 1 практически достигает величины скорости волны Стоунли. Из графиков смещений (скоростей), приведенных на рис. 3 , видно, что глубина проникновения этой моды в жидкость 
значительно больше глубины проникновения ее в упругое тело. При этом движения в этой моде при $\bar{h}=100$ происходят, главным образом, в жидком слое.

В работе [10] показано, что фазовая скорость и структура волны Стоунли - Шольте при взаимодействии твердого и жидкого полупространств зависят от механических параметров гидроупругой системы и определяются соотношением между скоростью волны звука в жидком и скоростью волны Рэлея в твердом полупространствах. При этом, если $\bar{a}_{0}>\bar{c}_{R}$, то глубина проникновения волны Стоунли в упругое полупространство больше глубины проникновения в жидкое полупространство. В случае выполнения условия $\bar{a}_{0}<\bar{c}_{R}$ глубина проникновения волны Стоунли в жидкость больше глубины проникания в твердое тело. В рассматриваемом случае механические параметры гидроупругой системы органическое стекло (менее жесткий материал) - вода таковы, что скорость распространения звуковой волны в жидкости $\bar{a}_{0}\left(\bar{a}_{0}=1,152595\right)$ больше скорости волны Рэлея $\bar{c}_{R}$ $\left(\bar{c}_{R}=0,933557\right)$. Для пары сталь (более жесткий материал) - вода имеет место соотношение $\bar{a}_{0}=0,463021<\bar{c}_{R}=0,923007$. Из графиков, приведенных на рис. 2 и 3, следует, что полученные результаты согласуются с выводами статьи [10]. Это свидетельствует о том, что эти закономерности волн характерны не только при взаимодействии жидкого и твердого полупространств, но также справедливы и для низшей поверхностной моды, распространяющейся в гидроупругой системе слой жидкости - упругое полупространство.

Заключение. Анализ полученных результатов показал, что низшая мода с увеличением частоты трансформируется в поверхностную волну Стоунли и ее локализация в средах зависит от соотношения между скоростью волны звука в жидком слое и скоростью волны Рэлея в упругом полупространстве. При этом в случае контакта жидкости с менее жестким материалом $\left(\bar{a}_{0}>\bar{c}_{R}\right)$ значительная часть потока энергии сосредотачивается в твердом теле. С уменьшением этого соотношения поток энергии перемещается из упругого тела в жидкий слой. При $\bar{a}_{0}<\bar{c}_{R}$ жидкий слой становится основным волноводом, по которому осуществляется перенос большей части волновой энергии, а вклад упругого тела в общий поток энергии становится незначительным.

\section{ЦИТИРОВАННАЯ ЛИТЕРАТУРА}

1. Викторов И.А. Звуковые поверхностные волны в твердых телах. Москва: Наука, 1981. 288 с.

2. Викторов И.А. К расчету фазовых скоростей поверхностных волн на границе твердого полупространства с жидким слоем. Акуст. журн. 1977. 23, № 6. С. 947-948.

3. Bagno A.M., Guz A.N. Elastic waves in pre-stressed bodies interacting with a fluid (survey). Int. Appl. Mech. 1997. 33, No 6. P. 435-463.

4. Guz A. N., Zhuk A. P., Bagno A. M. Dynamics of elastic bodies, solid particles, and fluid parcels in a compressible viscous fluid (review). Int. Appl. Mech. 2016. 52, No 5. P. 449-507.

5. Guz A. N. Aerohydroelasticity problems for bodies with initial stresses. Int. Appl. Mech. 1980. 16, No 3. P. $175-190$.

6. Гузь А.Н. Упругие волны в телах с начальными напряжениями: 2 тома. Киев: Наук. думка, 1986. Т. 2. 536 с.

7. Гузь А.Н. Упругие волны в телах с начальными (остаточными) напряжениями. Киев: А.С.К., 2004. 672 с.

8. Гузь А.Н. Динамика сжимаемой вязкой жидкости. Киев: А.С.К., 1998. 350 с.

9. Guz A.N. Dynamics of compressible viscous fluid. Cambridge: Cambridge Scientific Publishers, 2009. 428 p.

10. Волькенштейн М.М., Левин В.М. Структура волны Стоунли на границе вязкой жидкости и твердого тела. Акуст. журн. 1988. 34, № 4. С. 608-615.

Поступило в редакцию 21.02.2017 


\section{REFERENCES}

1. Viktorov, I. A. (1981). Sound surface waves in solids. Moscow: Nauka (in Russian).

2. Viktorov, I. A. (1977). To computation of phase velocities of surface waves on the interface of solid semi-space with a liquid layer. Acoustic J., 23, No. 6, pp. 947-948 (in Russian).

3. Bagno, A.M., Guz, A.N. (1997). Elastic waves in pre-stressed bodies interacting with a fluid (survey). Int. Appl. Mech., 33, No. 6, pp. 435-463.

4. Guz, A. N., Zhuk, A. P., Bagno, A. M. (2016). Dynamics of elastic bodies, solid particles, and fluid parcels in a compressible viscous fluid (review). Int. Appl. Mech., 52, No. 5, pp. 449-507.

5. Guz, A. N. (1980). Aerohydroelasticity problems for bodies with initial stresses. Int. Appl. Mech., 16, No. 3, pp. 175-190.

6. Guz, A. N. (1986). Elastic waves in bodies with initial stresses. 2 vols. Kiev: Naukova Dumka (in Russian).

7. Guz, A. N. (2004). Elastic waves in bodies with initial (residual) stresses. Kiev: A.S.K. (in Russian).

8. Guz, A. N. (1998). Dynamics of compressible viscous fluid. Kiev: A.S.K. (in Russian).

9. Guz, A. N. (2009). Dynamics of compressible viscous fluid. Cambridge: Cambridge Scientific Publishers.

10. Volkenstein, M. M. \& Levin, V. M. (1988). Structure of Stoneley wave on the boundary of a viscous liquid and a solid. Acoustic J., 34, No. 4, pp. 608-615 (in Russian).

Received 21.02.2017

\section{О.М. Багно}

Інститут механіки ім. С.П. Тимошенка НАН України, Київ

E-mail: alexbag2016@gmail.com

\section{ПРО ЛОКАЛІЗАЦІЮ ПОВЕРХНЕВИХ ХВИЛЬ У ШАРІ ІДЕАЛЬНОЇ СТИСЛИВОЇ РІДИНИ, ЩО ВЗАЄМОДІЕ 3 ПРУЖНИМ ПІВПРОСТОРОМ}

На основі тривимірних лінійних рівнянь класичної теорії пружності для твердого тіла та лінеаризованих рівнянь Ейлера для рідкого середовища досліджено поширення квазілембовських хвиль у системі пружний півпростір - шар ідеальної стисливої рідини. Побудовано дисперсійні криві для нормальних хвиль у широкому діапазоні частот. Проаналізовано вплив товщини шару ідеальної стисливої рідини на дисперсію фазових швидкостей квазілембовських мод у гідропружному хвилеводі. Досліджено властивості локалізації поверхневих хвиль у пружно-рідинних хвилеводах. Числові результати наведено у вигляді графіків та дано їх аналіз.

Ключові слова: дисперсія хвиль, фазова швидкість, пружний півпростір, шар ідеальної стисливої рідини, квазілембовські моди, локалізація поверхневих хвиль.

\section{O.M. Bahno}

S.P. Timoshenko Institute of Mechanics of the NAS of Ukraine, Kiev E-mail: alexbag2016@gmail.com

ON THE LOCALIZATION OF SURFACE WAVES IN THE LAYER OF AN IDEAL COMPRESSIBLE FLUID INTERACTING WITH THE ELASTIC HALF-SPACE

The propagation of quasi-Lamb waves in the system "layer of ideal compressible liquid - elastic half-space" is studied, by using the three-dimensional equations of the classical elasticity theory for a solid body and the linearized Euler equations for a fluid. The dispersion curves for normal waves over a wide range of frequencies are constructed. The influence of the thickness of the layer of an ideal compressible fluid on the dispersion of the phase velocity of quasi-Lamb modes in a hydroelastic waveguide is analyzed. The localization properties of the surface waves in elastic-fluid waveguides are studied. The numerical results obtained are presented in the form of plots and analyzed.

Keywords: dispersion of waves, phase velocity, elastic half-space, layer of an ideal compressible fluid, quasi-Lamb modes, localization of surface waves. 